\title{
Iridium-Induced Isomerization of 2-Substituted Pyridines to N-Heterocyclic Carbenes
}

Eleuterio Alvarez, Salvador Conejero, Margarita Paneque, Ana Petronilho, Manuel L. Poveda*, Oracio Serrano, Ernesto Carmona*

Instituto de Investigaciones Químicas, Departamento de Química Inorgánica, CSIC, Universidad de Sevilla, Avda. Américo Vespucio no 49, 41092 Sevilla, Spain

\section{Contents:}

General

Synthesis and spectroscopic and analytical data for new compounds

Table S.1. Crystal data and structure refinement for compound 2-Ph.

Table S.2. Crystal data and structure refinement for compound 2-Me.

Figure S.1. ORTEP representation of the molecular structure of compound 2-Ph.

Thermal ellipsoids plot (50 \% ellipsoid throughout).

General. Microanalyses were performed by the Microanalytical Service of the Instituto de Investigaciones Químicas (Sevilla, Spain). Infrared spectra were obtained from Bruker Vector 22 spectrometer. The NMR instruments were Bruker DRX-500, DRX-400 and 
DPX-300 spectrometers. Spectra were referenced to external $\mathrm{SiMe}_{4}(\delta 0 \mathrm{ppm})$ using the residual protio solvent peaks as internal standards ( ${ }^{1} \mathrm{H}$ NMR experiments) or the characteristic resonances of the solvent nuclei $\left({ }^{13} \mathrm{C}\right.$ NMR experiments). Spectral assignments were made by routine one- and two-dimensional NMR experiments where appropriate. All manipulations were performed under dry, oxygen-free dinitrogen, following conventional Schlenk techniques. The complex $\operatorname{Tp}{ }^{\mathrm{Me}} \operatorname{Ir}\left(\mathrm{C}_{6} \mathrm{H}_{5}\right)_{2} \mathrm{~N}_{2}$ was obtained by published procedures. ${ }^{1}$

General procedure for complexes 2: The corresponding pyridine (1.2 eq) was added, under nitrogen, to a solution of $0.2 \mathrm{~g}(0.3 \mathrm{mmol})$ of complex $1 \mathrm{in} 12 \mathrm{~mL}$ of $\mathrm{C}_{6} \mathrm{H}_{6}$. The mixture was heated at $90{ }^{\circ} \mathrm{C}$ during 2 hours (2-methylpyridine) or 4 hours (2phenylpyridine), and the solvent was then removed under vacuum. The products were purified by column chromatography on silica gel, using a mixture of solvents hexane:diethyl ether (9:1) as eluant for complex 2-Me or hexane:diethyl ether (40:1) for complex 2-Ph (all aromatic ${ }^{3} J_{\mathrm{HH}} \sim 7-8 \mathrm{~Hz}$ ).

2-Me

Yield: $45 \%$

IR (KBr): v(N-H) $3325 \mathrm{~cm}^{-1}$.

${ }^{1} \mathbf{H}\left(\mathrm{CD}_{2} \mathrm{Cl}_{2},-60{ }^{\circ} \mathrm{C}\right): \delta 12.91$ (br s, $\left.1 \mathrm{H}, \mathrm{NH}\right), 8.15\left(\mathrm{~d}, 2 \mathrm{H}, \mathrm{H}_{\mathrm{o}}\right), 7.02\left(\mathrm{t}, 2 \mathrm{H}, \mathrm{H}_{\mathrm{m}}\right), 6.95$ (d, 2 H, $\mathrm{H}_{\mathrm{py}}$ ), 6.72 (m, $3 \mathrm{H}, 2 \mathrm{H}_{\mathrm{p}}, \mathrm{H}_{\mathrm{py}}$ ), 6.50 (t, $2 \mathrm{H}, \mathrm{H}_{\mathrm{m}}$ ), 6.43 (d, $2 \mathrm{H}, \mathrm{H}_{\mathrm{o}}$ ), 5.68 and 5.67 (s,

\footnotetext{
${ }^{1}$ Guiérrez-Puebla,E.; Monge A.; Nicasio, M. C.; Pérez, P. J.; Poveda, M. L.; Carmona, E. Chem. Eur. J. 1998, 4, 2225.
} 
1:2 ratio, $3 \mathrm{H}, \mathrm{H}_{\mathrm{pz}}$ ), 2.51 (s, $3 \mathrm{H}, \mathrm{Me}_{\mathrm{py}}$ ), 2.46, 2.44, 0.75 and 0.70 (s, 1:2:2:1 ratio, $18 \mathrm{H}, 6$ $\left.\mathrm{Me}_{\mathrm{pz}}\right)$.

${ }^{13} \mathbf{C}\left\{{ }^{1} \mathbf{H}\right\}\left(\mathrm{CD}_{2} \mathrm{Cl}_{2},-60{ }^{\circ} \mathrm{C}\right): \delta 176.6(\mathrm{~s}, \mathrm{Ir}=\mathrm{C}), 151.0$ and 149.9 (s, 1:2 ratio, $\left.\mathrm{C}_{\mathrm{qpz}}\right), 148.2$ (s, $\mathrm{C}_{\mathrm{qpy}}$ ), 143.4 (s, $3 \mathrm{C}_{\mathrm{qpz}}$ ), 141.7 (s, $2 \mathrm{CH}_{\mathrm{o}}$ ), 140.2 (s, 2 Ir-C), 135.2 (s, $\mathrm{CH}_{\mathrm{py}}$ ), 134.5 (s, 2 $\mathrm{CH}_{\mathrm{o}}$ ), 126.3 and 126.2 (s, $4 \mathrm{CH}_{\mathrm{m}}$ ), $125.0\left(\mathrm{~s}, \mathrm{CH}_{\mathrm{py}}\right), 120.8\left(\mathrm{~s}, 2 \mathrm{CH}_{\mathrm{p}}\right), 116.0\left(\mathrm{~s}, \mathrm{CH}_{\mathrm{py}}\right)$, 107.2 and 107.0 (s, 1:2 ratio, $\mathrm{CH}_{\mathrm{pz}}$ ), 20.0 (s, $\mathrm{Me}_{\mathrm{py}}$ ), 14.1, 13.6 and 13.5 (s, 1:2:3 ratio, 6 $\left.\mathrm{Me}_{\mathrm{pz}}\right)$.

HRMS $\mathrm{m} / \mathrm{z}$ calcd. for $\mathrm{C}_{33} \mathrm{H}_{39} \mathrm{BIrN} \mathrm{N}_{7}$ (found): 737.303885 (737.298942)

2-Ph

Yield: $65 \%$

IR (KBr): v(N-H) $3325 \mathrm{~cm}^{-1}$.

${ }^{1} \mathbf{H}\left(\mathrm{CD}_{2} \mathrm{Cl}_{2},-60{ }^{\circ} \mathrm{C}\right): \delta 13.40$ (s, $\left.1 \mathrm{H}, \mathrm{NH}\right), 8.25\left(\mathrm{~d}, 2 \mathrm{H}, \mathrm{H}_{\mathrm{o}}\right), 7.60$ ( br s, $\left.5 \mathrm{H}, \mathrm{Ph}\right), 7.14$ (m, 3H, $\mathrm{H}_{\mathrm{py}}$ ), 7.00 (t, $2 \mathrm{H}, \mathrm{H}_{\mathrm{m}}$ ), 6.77 (t, $2 \mathrm{H}, \mathrm{H}_{\mathrm{p}}$ ), 6.55 (t, $2 \mathrm{H}, \mathrm{H}_{\mathrm{m}}$ ), $6.51\left(\mathrm{~d}, 2 \mathrm{H}, 2 \mathrm{H}_{\mathrm{o}}\right.$ ), 5.71 and 5.70 (s, 2:1 ratio, $3 \mathrm{H}, \mathrm{CH}_{\mathrm{pz}}$ ), 2.48, 2.47, 0.84 and 0.78 (s, $18 \mathrm{H}, 1: 2: 2: 1$ ratio, 6 $\left.\mathrm{Me}_{\mathrm{pz}}\right)$.

${ }^{13} \mathbf{C}\left\{{ }^{1} \mathbf{H}\right\}\left(\mathrm{CD}_{2} \mathrm{Cl}_{2},-60{ }^{\circ} \mathrm{C}\right): \delta 177.5$ (s, Ir=C), 151.1 and 150.2 (s, 1:2 ratio, $\mathrm{C}_{\mathrm{qpz}}$ ), 148.8 (s, $\mathrm{C}_{\mathrm{qpy}}$ ), 143.5 (s, $3 \mathrm{C}_{\mathrm{qpz}}$ ), 141.6 (s, $2 \mathrm{CH}_{\mathrm{o}}$ ), 140.7 (s, $\mathrm{CH}_{\mathrm{py}}$ ), 139.9 (s, 2 Ir-C), 135.2 (s, 2 $\mathrm{CH}_{\mathrm{o}}$ ), 135.1 (s, $\left.\mathrm{CH}_{\mathrm{py}}\right), 133.3$ (s, $\left.\mathrm{C}_{\mathrm{q} P h}\right), 131.3,130.2$ and 126.9 (s, 1:2:2 ratio, $\left.\mathrm{CH}_{\mathrm{Ph}}\right), 126.4$ 
and 126.3 (s, $4 \mathrm{CH}_{\mathrm{m}}$ ), $121.0\left(\mathrm{~s}, 2 \mathrm{CH}_{\mathrm{p}}\right), 114.8\left(\mathrm{~s}, \mathrm{CH}_{\mathrm{py}}\right), 107.3$ and 107.2 (s, 1:2 ratio, $\mathrm{CH}_{\mathrm{pz}}$ ), 14.2 and 13.5 (s, 1:5 ratio, $\left.6 \mathrm{Me}_{\mathrm{pz}}\right)$.

Anal. Calc. for $\mathrm{C}_{38} \mathrm{H}_{41} \mathrm{BIrN}_{7}$ : C, 57.10; H, 5.18; N, 12.27. Found: C, 57.2; H 5.2; N, 12.2.

HRMS m/z calcd. for $\mathrm{C}_{38} \mathrm{H}_{41} \mathrm{BIrN}_{7}$ (found): 799.21442 (799.214592).

\section{Compound 3-Me}

\section{Synthesis:}

The compound $\mathrm{Tp}^{\mathrm{Me} 2} \operatorname{Ir}(\mathrm{Ph})_{2} \mathrm{~N}_{2}(0.2 \mathrm{~g}, 0.3 \mathrm{mmol})$ was dissolved in $12 \mathrm{~mL}$ of $\mathrm{C}_{6} \mathrm{H}_{6}$, and 2picoline $(0.035 \mathrm{~mL}, 0.36 \mathrm{mmol})$ was then added. The solution was stirred at $60{ }^{\circ} \mathrm{C}$ for 2 hours and the solvent then evaporated under vacuum. The solid residue was washed with pentane $(2 \times 10 \mathrm{~mL})$ at $0{ }^{\circ} \mathrm{C}$, and the white-off solid dried in vacuo.

Yield: $50 \%$

${ }^{1} \mathbf{H}\left(\mathrm{CD}_{2} \mathrm{Cl}_{2},-60{ }^{\circ} \mathrm{C}\right): \delta 10.54\left(\mathrm{~d}, 1 \mathrm{H}, \mathrm{H}_{\mathrm{py}}\right), 8.24\left(\mathrm{~d}, 2 \mathrm{H}, \mathrm{H}_{\mathrm{o}}\right), 7.53\left(\mathrm{t}, 1 \mathrm{H}, \mathrm{H}_{\mathrm{py}}\right), 7.14$ (t, 1 H, $\mathrm{H}_{\mathrm{py}}$ ), 7.02 (t, $2 \mathrm{H}, \mathrm{H}_{\mathrm{m}}$ ), 6.92 (d, $1 \mathrm{H}, \mathrm{H}_{\mathrm{py}}$ ), 6.73 (t, $2 \mathrm{H}, \mathrm{H}_{\mathrm{p}}$ ), 6.48 (t, $2 \mathrm{H}, \mathrm{H}_{\mathrm{m}}$ ), 6.39 (d, $2 \mathrm{H}, \mathrm{H}_{\mathrm{o}}$ ), 5.71 and 5.54, (s, 1:2 ratio, $3 \mathrm{H}, \mathrm{H}_{\mathrm{pz}}$ ), 2.49 (s, $6 \mathrm{H}, 2 \mathrm{Me}_{\mathrm{pz}}$ ), 2.44 (s, $3 \mathrm{H}, \mathrm{Me}_{\mathrm{py}}$ ), 1.04, 0.73 and $0.46\left(\mathrm{~s}, 1: 2: 1\right.$ ratio, $\left.12 \mathrm{H}, 4 \mathrm{Me}_{\mathrm{pz}}\right)$.

${ }^{13} \mathbf{C}\left\{{ }^{1} \mathbf{H}\right\}\left(\mathrm{CD}_{2} \mathrm{Cl}_{2},-60{ }^{\circ} \mathrm{C}\right): \delta 165.1$ (s, $\left.\mathrm{C}_{\mathrm{qpy}}\right), 156.2\left(\mathrm{~s}, \mathrm{CH}_{\mathrm{py}}\right), 152.2$ and 150.6 (s, $1: 2$ ratio, $\mathrm{C}_{\mathrm{qpz}}$ ), 144.0 and 143.5 (s, 2:1 ratio, $\mathrm{C}_{\mathrm{qpz}}$ ), 142.6 (s, $2 \mathrm{CH}_{\mathrm{o}}$ ), 137.2 (s, $\left.2 \mathrm{Ir}-\mathrm{C}\right), 137.0$ (s, $\mathrm{CH}_{\mathrm{py}}$ ), 136.1 (s, $2 \mathrm{CH}_{\mathrm{o}}$ ), 128.1 (s, $\mathrm{CH}_{\mathrm{py}}$ ), 125.5 and 124.9 (s, $4 \mathrm{CH}_{\mathrm{m}}$ ), 122.1 (s, $\mathrm{CH}_{\mathrm{py}}$ ), 
$121.0\left(\mathrm{~s}, 2 \mathrm{CH}_{\mathrm{p}}\right), 107.7$ and 107.0 (s, 1:2 ratio, $\mathrm{CH}_{\mathrm{pz}}$ ), 22.6 (s, $\left.\mathrm{Me}_{\mathrm{py}}\right), 14.3,13.8,13.2$ and 12.8 (s, 1:1:2:2 ratio, $\left.6 \mathrm{Me}_{\mathrm{pz}}\right)$.

Anal. Calc. for $\mathrm{C}_{33} \mathrm{H}_{39} B \operatorname{IrN}_{7}$ : C, 53.80; H, 5.34; N, 13.31. Found: C, 53.8; H 5.4; N, 12.3.

\section{Compound 4-Ph}

\section{Synthesis:}

The compound $\mathrm{Tp}^{\mathrm{Me} 2} \mathrm{Ir}(\mathrm{Ph})_{2} \mathrm{~N}_{2}(0.2 \mathrm{~g}, 0.3 \mathrm{mmol})$ was dissolved in $12 \mathrm{~mL}$ of $\mathrm{C}_{6} \mathrm{H}_{6}$, and a $10^{-1} \mathrm{M}$ (in $\mathrm{C}_{6} \mathrm{H}_{6}$ ) solution of a 2-phenylpyridine (3.6 mL, $0.36 \mathrm{mmol}$ ) was added. The solution was stirred at $60{ }^{\circ} \mathrm{C}$ for $40 \mathrm{~min}$ and the solvent then evaporated under vacuum. The solid residue was purified by column chromatography on silica gel, using a mixture of hexane:diethylether (10:1) as eluant.

Yield: $55 \%$

IR (KBr): v(N-H) $3050 \mathrm{~cm}^{-1}$.

${ }^{1} \mathbf{H}\left(\mathrm{CD}_{2} \mathrm{Cl}_{2},-10{ }^{\circ} \mathrm{C}\right): \delta 8.03$ (d, $\left.2 \mathrm{H}, 2 \mathrm{H}_{\mathrm{o}-\mathrm{Phpy}}\right), 7.63$ (t, $\left.2 \mathrm{H}, 2 \mathrm{H}_{\mathrm{m}-\mathrm{Phpy}}\right), 7.57$ (t, $1 \mathrm{H}, \mathrm{H}_{\mathrm{p}^{-}}$ Phpy) 7.51 (t, $1 \mathrm{H}, \mathrm{H}_{\mathrm{o}}$ ), 7.20 (br s, $3 \mathrm{H}, \mathrm{H}_{\mathrm{py}}$ ), 6.99 (t, $1 \mathrm{H}, \mathrm{H}_{\mathrm{m}}$ ), 6.78 (t, $1 \mathrm{H}, \mathrm{H}_{\mathrm{p}}$ ), 6.66 (t, 1 $\mathrm{H}, \mathrm{H}_{\mathrm{m}}$ ), 6.38 (d, $1 \mathrm{H}, \mathrm{H}_{\mathrm{o}}$ ), 5.79, 5.69 and 5.61 (s, $1 \mathrm{H}$ each, $\mathrm{H}_{\mathrm{pz}}$ ), 2.49, 2.42, 2.39, 1.52, 1.49 and 1.30 (s, $3 \mathrm{H}$ each, $6 \mathrm{Me}_{\mathrm{pz}}$ ). 
${ }^{13} \mathrm{C}\left\{{ }^{1} \mathrm{H}\right\}\left(\mathrm{CD}_{2} \mathrm{Cl}_{2},-10{ }^{\circ} \mathrm{C}\right): \delta 171.9(\mathrm{~s}, \mathrm{Ir}=\mathrm{C}), 150.7,150.6$ and $150.2\left(\mathrm{~s}, 3 \mathrm{C}_{\mathrm{qpz}}\right), 147.3$ (s, $\mathrm{C}_{\mathrm{qpy}}$ ), 143.4, 142.9 and 142.8 (s, $3 \mathrm{C}_{\mathrm{qpz}}$ ), $140.9\left(\mathrm{~s}, \mathrm{CH}_{\mathrm{o}}\right), 137.1\left(\mathrm{~s}, \mathrm{CH}_{\mathrm{py}}\right), 136.7$ (s, IrC), 134.9 (s, $\left.\mathrm{CH}_{\mathrm{py}}\right), 133.9$ (s, $\mathrm{C}_{\mathrm{qPhpy}}$ ), 130.6 and 130.6 (s, $\left.\mathrm{CH}_{0}, \mathrm{CH}_{\mathrm{p}-\mathrm{Phpy}}\right), 129.9$ (s, $2 \mathrm{CH}_{\mathrm{m}-}$ Phpy), 126.3 (s, $2 \mathrm{CH}_{\text {o-Phpy }}$ ), 126.2 and $125.0\left(\mathrm{~s}, \mathrm{CH}_{\mathrm{m}}\right), 121.3$ (s, $\left.\mathrm{CH}_{\mathrm{p}}\right), 114.1\left(\mathrm{~s}, \mathrm{CH}_{\mathrm{py}}\right)$, 107.3, 107.1 and 107.0 (s, $\left.3 \mathrm{CH}_{\mathrm{pz}}\right), 16.3,13.4,13.2,12.0,12.8$ and $12.0\left(\mathrm{~s}, 6 \mathrm{Me}_{\mathrm{pz}}\right)$. 
Table S.1. Crystal data and structure refinement for 2-Ph.

\begin{tabular}{|c|c|c|}
\hline Empirical formula & \multicolumn{2}{|c|}{$\begin{array}{rl}\mathrm{C}_{39} \mathrm{H}_{43} & \mathrm{~B} \mathrm{Cl}_{2} \mathrm{Ir} \mathrm{N}_{7} \\
& {\left[\mathrm{C}_{38} \mathrm{H}_{41} \mathrm{~B} \text { Ir N } \mathrm{N}_{7}, \mathrm{C} \mathrm{H}_{2} \mathrm{Cl}_{2}\right]}\end{array}$} \\
\hline Formula weight & \multicolumn{2}{|c|}{883.71} \\
\hline Temperature & \multicolumn{2}{|l|}{$100(2) \mathrm{K}$} \\
\hline Wavelength & \multicolumn{2}{|l|}{$0.71073 \AA$} \\
\hline Crystal system & \multicolumn{2}{|l|}{ Monoclinic } \\
\hline Space group & \multicolumn{2}{|l|}{$\mathrm{C} 2 / \mathrm{c}$} \\
\hline \multirow[t]{3}{*}{ Unit cell dimensions } & $\mathrm{a}=40.2432(8) \AA$ & $\alpha=90^{\circ}$ \\
\hline & $\mathrm{b}=10.1628(2) \AA$ & $\beta=101.4560(10)^{\circ}$ \\
\hline & $c=18.6214(4) \AA$ & $\gamma=90^{\circ}$ \\
\hline Volume & \multicolumn{2}{|l|}{$7464.1(3) \AA^{3}$} \\
\hline $\mathrm{Z}$ & \multicolumn{2}{|l|}{8} \\
\hline Density (calculated) & \multicolumn{2}{|l|}{$1.573 \mathrm{Mg} / \mathrm{m}^{3}$} \\
\hline Absorption coefficient & \multicolumn{2}{|l|}{$3.759 \mathrm{~mm}^{-1}$} \\
\hline $\mathrm{F}(000)$ & \multicolumn{2}{|l|}{3536} \\
\hline Crystal size & \multicolumn{2}{|c|}{$0.27 \times 0.20 \times 0.18 \mathrm{~mm}^{3}$} \\
\hline Theta range for data collection & \multicolumn{2}{|c|}{2.89 to $30.57^{\circ}$} \\
\hline Index ranges & \multicolumn{2}{|c|}{$-57<=\mathrm{h}<=57,-14<=\mathrm{k}<=14,-26<=\mathrm{l}<=26$} \\
\hline Reflections collected & \multicolumn{2}{|c|}{69220} \\
\hline Independent reflections & \multicolumn{2}{|c|}{$11433[\mathrm{R}$ (int) $=0.0417]$} \\
\hline Completeness to theta $=30.57^{\circ}$ & \multicolumn{2}{|l|}{$99.6 \%$} \\
\hline Absorption correction & \multicolumn{2}{|c|}{ Semi-empirical from equivalents } \\
\hline Max. and min. transmission & \multicolumn{2}{|l|}{0.5510 and 0.4301} \\
\hline Refinement method & \multicolumn{2}{|c|}{ Full-matrix least-squares on $\mathrm{F}^{2}$} \\
\hline Data / restraints / parameters & \multicolumn{2}{|l|}{11433 / 16 / 479} \\
\hline Goodness-of-fit on $\mathrm{F}^{2}$ & \multicolumn{2}{|l|}{1.084} \\
\hline Final R indices [I>2sigma(I)] & \multicolumn{2}{|c|}{$\mathrm{R} 1=0.0296, \mathrm{wR} 2=0.0601$} \\
\hline $\mathrm{R}$ indices (all data) & \multicolumn{2}{|c|}{$\mathrm{R} 1=0.0437, \mathrm{wR} 2=0.0649$} \\
\hline Largest diff. peak and hole & \multicolumn{2}{|c|}{1.020 and -2.505 e. $\AA^{-3}$} \\
\hline
\end{tabular}


Table S.2. Crystal data and structure refinement for 2-Me.

\begin{tabular}{|c|c|c|}
\hline Empirical formula & \multicolumn{2}{|c|}{$\mathrm{C}_{71} \mathrm{H}_{90} \mathrm{~B}_{2} \mathrm{Ir}_{2} \mathrm{~N}_{14}$} \\
\hline Formula weight & \multicolumn{2}{|l|}{1545.59} \\
\hline Temperature & \multicolumn{2}{|l|}{$100(2) \mathrm{K}$} \\
\hline Wavelength & \multicolumn{2}{|l|}{$0.71073 \AA$} \\
\hline Crystal system & \multicolumn{2}{|l|}{ Monoclinic } \\
\hline Space group & \multicolumn{2}{|l|}{$\mathrm{P} 2(1) / \mathrm{n}$} \\
\hline \multirow[t]{3}{*}{ Unit cell dimensions } & $\mathrm{a}=10.3293(2) \AA$ & $\alpha=90^{\circ}$. \\
\hline & $\mathrm{b}=25.2058(5) \AA$ & $\beta=99.9410(10)^{\circ}$. \\
\hline & $c=13.1798(3) \AA$ & $\gamma=90^{\circ}$ \\
\hline Volume & \multicolumn{2}{|l|}{ 3379.95(12) $\AA^{3}$} \\
\hline $\mathrm{Z}$ & \multicolumn{2}{|l|}{2} \\
\hline Density (calculated) & \multicolumn{2}{|l|}{$1.519 \mathrm{Mg} / \mathrm{m}^{3}$} \\
\hline Absorption coefficient & \multicolumn{2}{|l|}{$3.986 \mathrm{~mm}^{-1}$} \\
\hline $\mathrm{F}(000)$ & \multicolumn{2}{|l|}{1556} \\
\hline Crystal size & \multicolumn{2}{|c|}{$0.29 \times 0.26 \times 0.20 \mathrm{~mm}^{3}$} \\
\hline Theta range for data collection & \multicolumn{2}{|l|}{2.86 to $30.51^{\circ}$. } \\
\hline Index ranges & \multicolumn{2}{|c|}{$-14<=\mathrm{h}<=14,-35<=\mathrm{k}<=35,-17<=\mathrm{l}<=18$} \\
\hline Reflections collected & \multicolumn{2}{|l|}{60903} \\
\hline Independent reflections & \multicolumn{2}{|c|}{$10242[\mathrm{R}(\mathrm{int})=0.0255]$} \\
\hline Completeness to theta $=30.51^{\circ}$ & \multicolumn{2}{|l|}{$99.4 \%$} \\
\hline Absorption correction & \multicolumn{2}{|c|}{ Semi-empirical from equivalents } \\
\hline Max. and min. transmission & \multicolumn{2}{|l|}{0.5028 and 0.3911} \\
\hline Refinement method & \multicolumn{2}{|c|}{ Full-matrix least-squares on $\mathrm{F}^{2}$} \\
\hline Data / restraints / parameters & \multicolumn{2}{|l|}{10242 / 56 / 427} \\
\hline Goodness-of-fit on $\mathrm{F}^{2}$ & \multicolumn{2}{|l|}{1.040} \\
\hline Final R indices [I $>2 \operatorname{sigma}(\mathrm{I})]$ & \multicolumn{2}{|c|}{$\mathrm{R} 1=0.0177, \mathrm{wR} 2=0.0403$} \\
\hline $\mathrm{R}$ indices (all data) & \multicolumn{2}{|c|}{$\mathrm{R} 1=0.0219, \mathrm{wR} 2=0.0416$} \\
\hline Largest diff. peak and hole & \multicolumn{2}{|c|}{0.762 and -0.629 e..$\AA^{-3}$} \\
\hline
\end{tabular}


Figure S.1. Thermal ellipsoid plot (50 \% ellipsoids) of compound 2-Ph. H atoms are omitted for clarity.

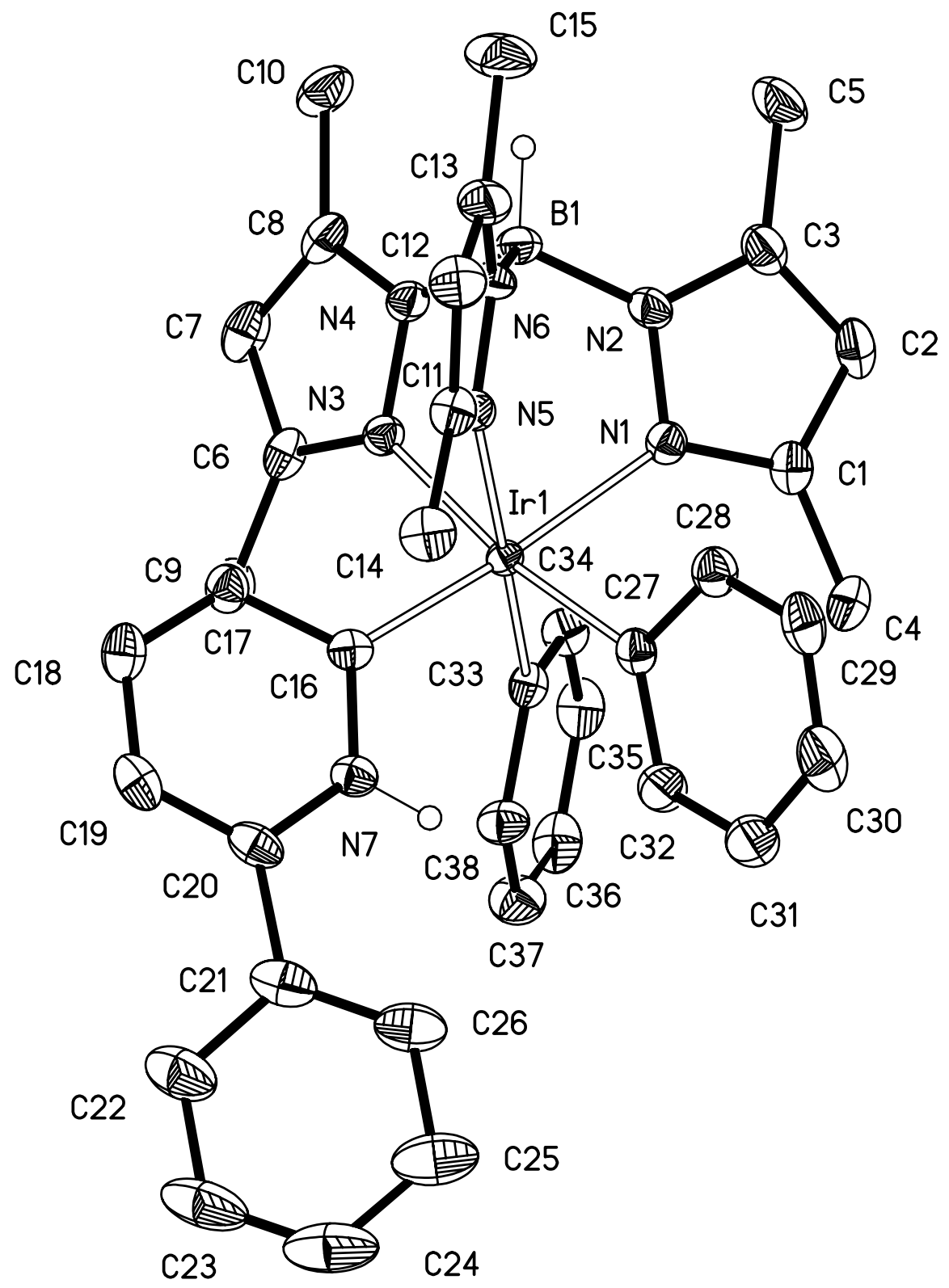

